\title{
La relación entre la captación de ingresos fiscales municipales y los recursos derivados de fuentes no tributarias tanto locales como externas en México
}

\section{The relationship between the collection of municipal tax revenues and resources derived from both local and external non-tax sources in Mexico}

\author{
VALENZUELA-REYNAGA, Rodolfo*† \& MORENO-MILLANES, María Dolores \\ Instituto Tecnológico de Sonora. Departamento de Contaduría y Finanzas. Calle 5 de Febrero 818, Centro, Urb. No. 1, \\ 85000 Cd Obregón, Son.
}

ID $1^{\text {er }}$ Autor: Rodolfo, Valenzuela-Reynaga / ORC ID: 0000-0003-3408-361X, CVU CONACYT ID: 287601

ID $2^{\text {do }}$ Coautor: María Dolores, Moreno-Millanes / ORC ID: 0000-0003-0772-2930, CVU CONACYT ID: 625356

DOI: $10.35429 / J L A .2019 .7 .3 .8 .16$

Recibido 05 de Enero, 2019; Aceptado 19 de Marzo, 2019

\begin{abstract}
Resumen
Los ingresos públicos municipales se clasifican en tributarios y no tributarios. En este trabajo se estudia la relación que existe entre los ingresos que los municipios captan a través de impuestos y el resto de los componentes que integran los ingresos totales para establecer en qué medida la obtención de recursos cuyo origen es de origen no impositivo afecta la generación de ingresos fiscales locales. Se emplea un panel de datos conformado por 310 observaciones para cada variable y se utiliza un modelo de regresión múltiple con efectos fijos considerando información financiera de los 31 municipios que son capitales de las entidades federativas en México para el período 2008 - 2017. Los resultados sugieren que los recursos provenientes de las transferencias federales y estatales tienen un impacto negativo en los niveles de ingresos obtenidos a través de impuestos municipales. Asimismo, la relación negativa localizada entre la contratación de deuda pública y los recursos fiscales propios podría suponer un relajamiento de la política recaudatoria municipal. Finalmente, los datos de otros ingresos derivados de fuentes locales de origen no impositivo como los derechos, productos y aprovechamientos muestran un efecto negativo en la captación de ingresos provenientes de la recaudación de impuestos.
\end{abstract}

Ingresos municipales, impuestos locales, finanzas públicas

\begin{abstract}
Municipal public revenues are classified as tax and nontax. In this work, the relationship between the income that the municipalities receive through taxes and the rest of the components that integrate the total income is studied to establish to what extent the obtaining of resources whose origin is of non-tax origin affects the generation of local tax revenue. A data panel consisting of 310observations is used for each variable and a multiple regression model with fixed effects is used considering financial information of the 31 municipalities that are capitals of the federal entities in Mexico for the period 2008 - 2017. The results suggest that resources from federal and state transfers have a negative impact on the income levels obtained through municipal taxes. Likewise, the negative localized relationship between the contracting of public debt and the own fiscal resources could suppose a relaxation of the municipal collection policy. Finally, data on other income derived from local sources of non-tax origin such as rights, products and uses show a negative effect on the collection of income from tax collection.
\end{abstract}

Municipal income, local taxes, public finances

Citación: VALENZUELA-REYNAGA, Rodolfo \& MORENO-MILLANES, María Dolores. La relación entre la captación de ingresos fiscales municipales y los recursos derivados de fuentes no tributarias tanto locales como externas en México. Revista de Aplicaciones del Derecho. 2019. 3-7: 8-16.

\footnotetext{
*Correspondencia al Autor (Correo Electrónico: rodolfo.valenzuela.reynaga@ gmail.com)

$\dagger$ Investigador contribuyendo como primer autor.
} 


\section{Introducción}

En el caso mexicano, los municipios representan el orden de gobierno de mayor proximidad con la población en términos geográficos. También es, sin duda, la instancia gubernamental que mejor conocimiento podría tener respecto de las necesidades fundamentales de los ciudadanos en cuanto a la provisión de bienes públicos básicos como los servicios de agua potable, acondicionamiento de vialidades, recolección de basura, entre otros.

Para el cumplimiento de sus atribuciones, facultades y obligaciones los municipios deben gestionar recursos financieros a través de diversos mecanismos provistos en las legislaciones tanto federales como locales. Por tanto, la administración de los ingresos, los egresos y la deuda pública constituye un aspecto esencial respecto del logro de los planes, programas y proyectos propuestos por cualquier gobierno municipal.

Los ingresos públicos locales se componen de recursos propios y de aquellos que provienen de fuentes ajenas al ámbito municipal (transferencias federales y estales así como los créditos u obligaciones financieras contraídas mediante operaciones de financiamiento). Con relación a los ingresos públicos propios estos pueden ser de tipo tributario y no tributario; los primeros se refieren a los recursos que se captan mediante la recaudación de impuestos locales mientras que los segundos no tienen carácter fiscal o impositivo.

Un reto fundamental de las finanzas públicas locales está vinculado con incrementar la generación de ingresos locales. En ese sentido, según datos de la Organización para la Cooperación y el Desarrollo Económico en el año 2016 la recaudación tributaria municipal en México representó solamente el $0.3 \%$ del Producto Interno Bruto (OCDE, 2017).

El objetivo de este trabajo es analizar la relación que existe entre el nivel de ingresos fiscales, es decir los que se originan a partir del cobro de impuestos, y los recursos no tributarios, tanto propios como externos al ámbito local (transferencias federales y estatales así como ingresos provenientes de la contratación de deuda pública).
En el análisis se consideran 31 municipios que, a su vez, son las capitales de las entidades federativas mexicanas.

La pertinencia del estudio estriba, fundamentalmente, en la identificación de factores que podrían afectar la generación de ingresos fiscales locales. Esto es, en la medida en que se robustece el debate académico y político en materia de finanzas públicas municipales se podrán diseñar políticas fiscales que fortalezcan las capacidades de los municipios respecto de la provisión de bienes y servicios de mejor calidad.

La hipótesis central de investigación sugiere una relación negativa entre el nivel de ingresos fiscales municipales y los recursos propios no tributarios así como los ingresos provenientes de fuentes externas (transferencias y financiamiento).

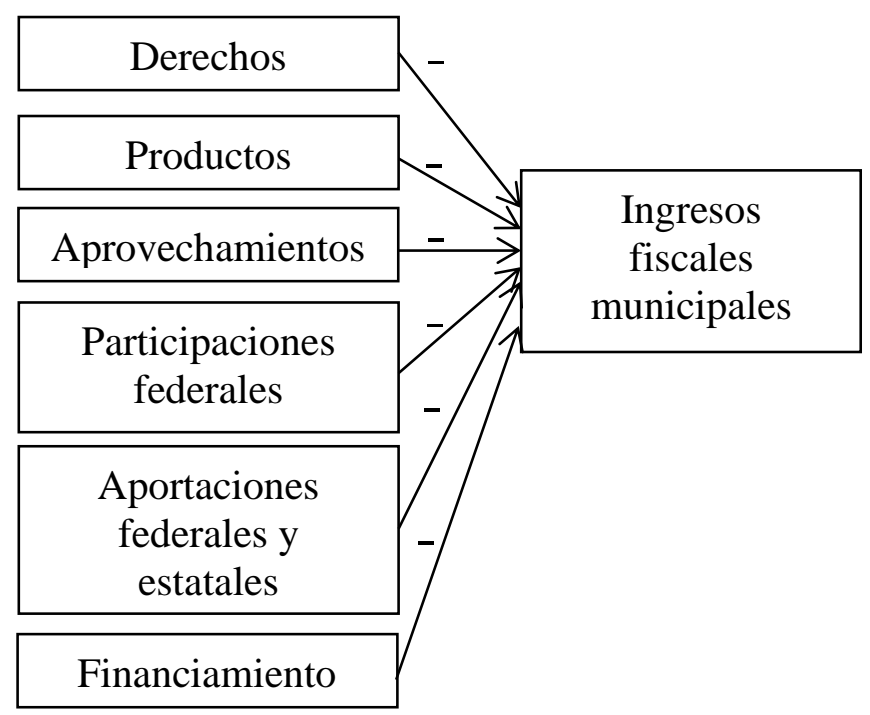

\section{Figura 1}

El documento se integra, inicialmente, por una sección introductoria en la cual se precisa el propósito central de este trabajo así como los elementos que permiten establecer la naturaleza y dimensión del problema de investigación. Posteriormente, se agrega un apartado en el que se expone el soporte teórico que da fundamento al estudio. Después se incorpora la sección en que se describe la estrategia metodológica empleada para el desarrollo de este trabajo. Enseguida se presenta la sección de resultados en la que se muestran los hallazgos localizados en la investigación. Finalmente, hay un apartado en el que se plantean las conclusiones del estudio. 


\section{Marco Teórico}

Para Londoño \& Ossa (2008) los funcionarios de las administraciones públicas municipales tienden a conducirse de la mejor forma posible o, en cierto modo, a mostrar un apropiado desempeño en sus labores con el propósito de enviar señales a la comunidad con relación a quiénes podrían ser los candidatos que podrían sucederles en sus encargos, dejando de lado, en cierto modo, aspectos políticos y alineándose al ámbito de la gerencia pública local.

Con relación a los ingresos municipales, al igual que en el caso del resto de los órdenes gubernamentales (estatal y federal) el ordenamiento legal que permite a los municipios allegarse de los recursos que serán utilizados para financiar el gasto público previsto en el presupuesto de egresos es la ley de ingresos municipales; esta norma dota de las atribuciones y facultades necesarias a las autoridades locales para establecer montos, tasas y tarifas relacionados con los ingresos tributarios y no tributarios que se habrán de obtener durante el ejercicio fiscal correspondiente (Núñez, 2005).

Mandujano (2010) plantea que, de acuerdo con la fracción IV del artículo 115 de la Constitución Política de los Estados Unidos Mexicanos, los municipios podrán administrar libremente su hacienda pública, entendida ésta como el total de recursos y bienes con que se cuentan así como la manera en que son administrados y ejercidos para conseguir los propósitos de la administración pública municipal. Los ingresos públicos de carácter fiscal están conformados por los recursos financieros que los municipios obtienen a través de la recaudación de impuestos (Cejudo \& Ríos, 2009).

Una clasificación inicial de los tipos de ingresos públicos es aquella que los agrupa en tributarios y no tributarios. En el primer grupo se encuentran los que provienen de impuestos locales y que una de las características esenciales es la obligatoriedad de los habitantes respecto de su pago; en el segundo grupo se encuentran aquellos que no tienen una naturaleza impositiva pero que forman parte del pago por algún tipo de contraprestación (Eguino, 2002).
En un trabajo realizado por Espinosa, Martinez \& Martell (2018) pretenden identificar por qué algunos municipios mexicanos son más eficaces en términos de la obtención de ingresos fiscales mediante la recaudación del impuesto predial que otros municipios. Para ello emplean un panel de datos considerando el período 2008 - 2013 y utilizan un modelo de regresión con efectos fijos. Los resultados que obtienen demuestran que las condiciones socioeconómicas de los municipios, las transferencias del resto de los órdenes de gobierno (estatal y federal), la actualización de los catastros municipales así como la uniformidad en los esquemas de gestión administrativa son factores que estarían explicando las diferencias que existen entre los municipios con relación a su capacidad y eficacia en el cobro del predial, el cual es el principal impuesto de orden municipal.

Empleando datos de panel para el período 1969 - 2010 con el propósito de estudiar el comportamiento de la recaudación del impuesto predial en México, Unda \& Moreno (2015) encuentran que un factor determinante con relación a la obtención de ingresos públicos locales a través de esa fuente de recursos es el área geográfica (la recaudación es más eficaz en zonas urbanas que en los municipios rurales).

Sour (2004) localiza que para el caso mexicano las transferencias, tanto condicionadas como las no condicionadas, han tenido un efecto negativo en las haciendas públicas municipales al ocasionar una disminución en la generación de ingresos propios. Señala, además, que el esfuerzo fiscal municipal se ha visto afectado en mayor medida en los municipios grandes que en los pequeños.

Con relación a factores de orden político (Chávez, 2014) localiza que existen algunos elementos que podrían explicar el desistimiento de los municipios mexicanos con relación al cobro del impuesto predial; encuentra que los municipios que cuentan con una elevada proporción de propiedad comunal son más proclives a no establecer medidas eficaces respecto de la obtención de ingresos fiscales a través del cobro del impuesto predial. Además, que esas posturas de desistimiento por parte de los municipios están vinculadas con mecanismos de que les permitan ganar elecciones. 
En cuanto a los ingresos que los municipios obtienen a través de la contratación de deuda pública o financiamiento, Ramíez \& Poom (2013) encuentran que el fenómeno político de gobiernos divididos ha tenido un impacto relevante en la disminución del nivel de endeudamiento municipal. Esto es, en la medida en que los procesos democratizadores permiten la alternancia política en las administraciones municipales es posible que se generen mejores incentivos para generar una cantidad mayor de ingresos propios y recurrir menos a la contratación de pasivos financieros.

A continuación, se definen literalmente, de acuerdo al clasificador por rubro de ingresos de la Ley General de Contabilidad Gubernamental, los capítulos de ingresos que habrán de ser estudiados en el presente trabajo.

- Impuestos. Son las contribuciones establecidas en Ley que deben pagar las personas físicas y morales que se encuentran en la situación jurídica o de hecho prevista por la misma y que sean distintas de las aportaciones de seguridad social, contribuciones de mejoras y derechos.

- Derechos. Son las contribuciones establecidas en Ley por el uso o aprovechamiento de los bienes del dominio público, así como por recibir servicios que presta el Estado en sus funciones de derecho público, excepto cuando se presten por organismos descentralizados $\mathrm{u}$ órganos desconcentrados cuando en este último caso, se trate de contraprestaciones que no se encuentren previstas en las leyes correspondientes. También son derechos las contribuciones a cargo de los organismos públicos descentralizados por prestar servicios exclusivos del Estado.

- Productos. Son los ingresos por contraprestaciones por los servicios que preste el Estado en sus funciones de derecho privado tales como los intereses que generan las cuentas bancarias de los entes públicos, entre otros, de conformidad con la legislación aplicable en la materia.
Aprovechamientos. Son los ingresos que percibe el Estado por funciones de derecho público distintos de: las contribuciones, los ingresos derivados de financiamientos y de los que obtengan los organismos descentralizados y las empresas de participación estatal y municipal.

- Participaciones federales. Son los ingresos que reciben los municipios que se derivan de la adhesión al Sistema Nacional de Coordinación Fiscal, así como las que correspondan a sistemas estatales de coordinación fiscal, determinados por las leyes correspondientes.

- $\quad$ Aportaciones estatales y federales. Son los ingresos que reciben los municipios previstos en la Ley de Coordinación Fiscal, cuyo gasto está condicionado a la consecución y cumplimiento de los objetivos que para cada tipo de aportación establece la legislación aplicable en la materia.

- Financiamiento. Son los ingresos obtenidos por la celebración de empréstitos internos o externos, a corto o largo plazo, aprobados en términos de la legislación correspondiente.

\section{Metodología}

El estudio es de tipo cuantitativo y de carácter explicativo. Dado que no existe un mecanismo de control sobre las variables involucradas en la investigación, el diseño es no experimental.

Asumiendo que para el cumplimiento del objetivo del estudio es conveniente considerar la temporalidad, la investigación es longitudinal; sin embargo, en virtud de que se incorpora la perspectiva transversal ya que se realizan mediciones en las variables para cada uno de los elementos individuales de la unidad de análisis se considera la construcción de un panel de datos con 310 observaciones para cada variable.

El período de estudio es de diez años (2008 - 2017). Se utiliza información sobre los ingresos públicos municipales y sus componentes. 
Mediante un modelo de regresión lineal múltiple con efectos fijos se estima la siguiente ecuación.

$\mathrm{IFM}_{\text {it }}=\mathrm{PF}_{\mathrm{it}}+\mathrm{AFE}_{\mathrm{it}}+\mathrm{P}_{\mathrm{it}}+\mathrm{D}_{\mathrm{it}}+\mathrm{A}_{\mathrm{it}}+\mathrm{F}_{\mathrm{it}}+\varepsilon_{\mathrm{it}}$

Donde:

IFM $=$ Ingresos fiscales municipales

$\mathrm{PF}=$ Participaciones federales

$\mathrm{AFE}=$ Aportaciones federales y estatales

$\mathrm{P}=$ Productos

$\mathrm{D}=$ Derechos

$\mathrm{A}=$ Aprovechamientos

$\mathrm{F}=$ Financiamiento

$\mathrm{i}=$ Municipio $1,2,3, \ldots, 31$

$\mathrm{t}=$ Año $1,2,3, \ldots, 10$

\section{Resultados}

Este apartado muestra los hallazgos localizados en el estudio y para tal propósito se organiza de la siguiente manera. Inicialmente se describe la evolución, en términos de tendencias o crecimiento porcentual, de cada uno de los capítulos de ingresos municipales durante el período de estudio. Enseguida se da cuenta del comportamiento tanto de los ingresos fiscales municipales como de los recursos transferidos por los órdenes estatales y federal con relación a los ingresos totales locales. Finalmente se agregan los datos que permiten determinar el cumplimiento, o no, de las hipótesis causales propuestas en la primera sección del documento.

Entre los años 2008 y 2017 la tasa de crecimiento, promedio anual, de los ingresos totales municipales fue de $6.9 \%$. Con relación a los recursos captados a través de la recaudación de impuestos se encontró una variación media de $8.9 \%$. Para el caso de los derechos, productos y aprovechamientos el ajuste fue de $10.6 \%, 29.3 \%$ y $15.6 \%$ respectivamente. En cuanto a los ingresos cuyo origen son las transferencias, se localizó un aumento del $7.6 \%$ en las participaciones federales y $12.4 \%$ en las aportaciones federales y estatales.

En el Gráfico 1 se observa que Puebla, Querétaro y San Luis Potosí fueron los tres municipios con la mayor variación porcentual, promedio anual, en cuanto a la generación de ingresos fiscales durante el período de estudio.
Por el contrario, La Paz, Xalapa y Mexicali mostraron tasas de crecimiento, que aunque positivas, resultaron inferiores al resto de las haciendas públicas locales. Cabe señalar que otros capítulos de ingresos locales no tributarios reportaron ajustes negativos en el período de estudio. En el apartado de derechos destaca el municipio de La Paz con $-2.8 \%$. En los recursos provenientes de productos se localizó un decrecimiento en Cuernavaca y Centro con $-12.8 \%$ y $-2.0 \%$ respectivamente. En cuanto a los aprovechamientos las disminuciones se observan en Centro $(-5.7 \%)$, Morelia (-4.2\%), Chilpancingo (-2.3\%) y en La Paz (-1.0\%).

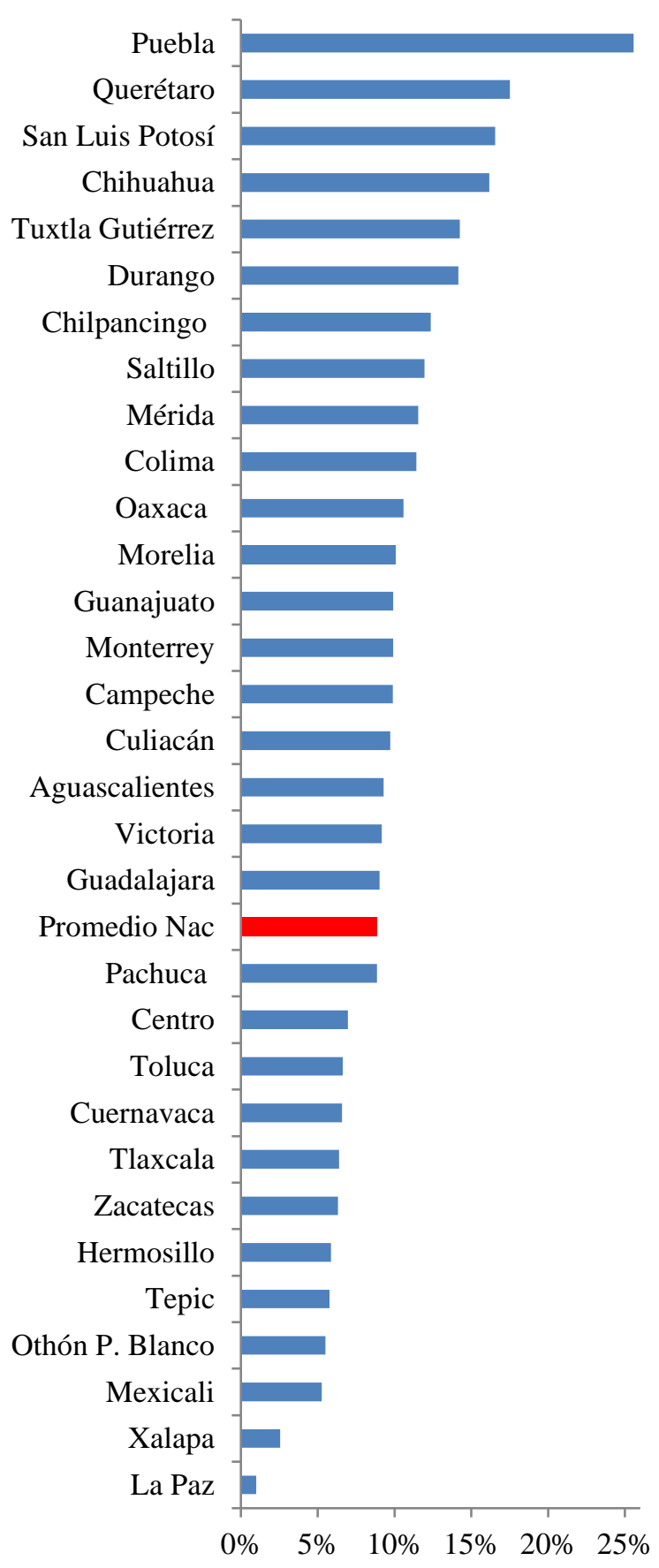

Gráfico 1 Crecimiento promedio anual de los ingresos fiscales locales en el período 2008 - 2017

Fuente: elaboración propia con datos de INEGI (2019)

VALENZUELA REYNAGA, Rodolfo \& MORENO MILLANES, María Dolores. La relación entre la captación de ingresos fiscales municipales y los recursos derivados de fuentes no tributarias tanto locales como externas en México. Revista de Aplicaciones del Derecho. 2019 
En lo concerniente a la importancia relativa de los ingresos fiscales y de las transferencias en el total de ingresos municipales se encontró que, en promedio, 15 centavos de cada peso tienen su origen en fuentes tributarias locales.

Por su parte, los recursos de libre disposición o que no tienen un propósito de gasto específico (participaciones federales) constituyeron el $34.1 \%$ de los ingresos totales, mientras que las transferencias recibidas por otros órdenes de gobierno (estatal y federal) y que si tienen un ejercicio presupuestal condicionado representaron el $28.9 \%$ de los ingresos totales municipales.

En los diez años que comprende el período de análisis no se observan variaciones significativas respecto de la composición, promedio, de la estructura de los ingresos públicos municipales (ver Tabla 1).

\begin{tabular}{|l|r|r|r|}
\hline \multicolumn{1}{|c}{ A ño } & Impuestos & \multicolumn{1}{c}{ Part_Fed } & Aport_Fed_Est \\
\hline 2008 & $13.5 \%$ & $36.0 \%$ & $26.7 \%$ \\
\hline 2009 & $13.2 \%$ & $33.4 \%$ & $28.0 \%$ \\
\hline 2010 & $13.3 \%$ & $33.1 \%$ & $29.8 \%$ \\
\hline 2011 & $12.3 \%$ & $33.0 \%$ & $29.1 \%$ \\
\hline 2012 & $14.8 \%$ & $31.3 \%$ & $27.0 \%$ \\
\hline 2013 & $15.1 \%$ & $34.7 \%$ & $27.1 \%$ \\
\hline 2014 & $15.6 \%$ & $33.7 \%$ & $29.7 \%$ \\
\hline 2015 & $16.0 \%$ & $32.0 \%$ & $29.2 \%$ \\
\hline 2016 & $17.6 \%$ & $35.7 \%$ & $30.7 \%$ \\
\hline 2017 & $16.6 \%$ & $34.1 \%$ & $26.7 \%$ \\
\hline
\end{tabular}

Tabla 1 Participación porcentual de los ingresos captados vía impuestos y transferencias federales en los ingresos totales

Fuente: elaboración propia con datos de INEGI (2019)

Se encuentra que en el período de estudio, de los ingresos municipales totales, $62.1 \%$ corresponden a participaciones federales $\mathrm{y}$ aportaciones federales y estatales.

Esto revela, sin duda, la ausencia de ajustes estructurales al modelo fiscal en el ámbito del federalismo y la descentralización hacendaria dado que no se aprecia algún indicio sobre la diminución que en materia de dependencia financiera presentan los municipios mexicanos con relación a los recursos que les son transferidos por los restantes órdenes gubernamentales.

No obstante, lo anterior, cuando se realiza el análisis individual de los municipios que forman parte del estudio es posible ubicar escenarios diversos.
Con relación a los ingresos públicos locales cuyo origen es de carácter fiscal (impuestos) se identifican 11 municipios en los cuales este tipo de recursos es menor al $10 \%$ de los ingresos totales municipales; estos son Tepic, Oaxaca, Campeche, Chilpancigo, Tuxtla Gutiérrez, Centro, Tlaxcala, Zacatecas, Guanajuato, Victoria y Othón P. Blanco. En contraste, para las haciendas públicas de los municipios de Querétaro, Chihuahua, Cuernavaca, Monterrey, Culiacán, Hermosillo y Mérida los recursos tributarios representan más de una quinta parte de los ingresos totales (ver Gráfico 2).

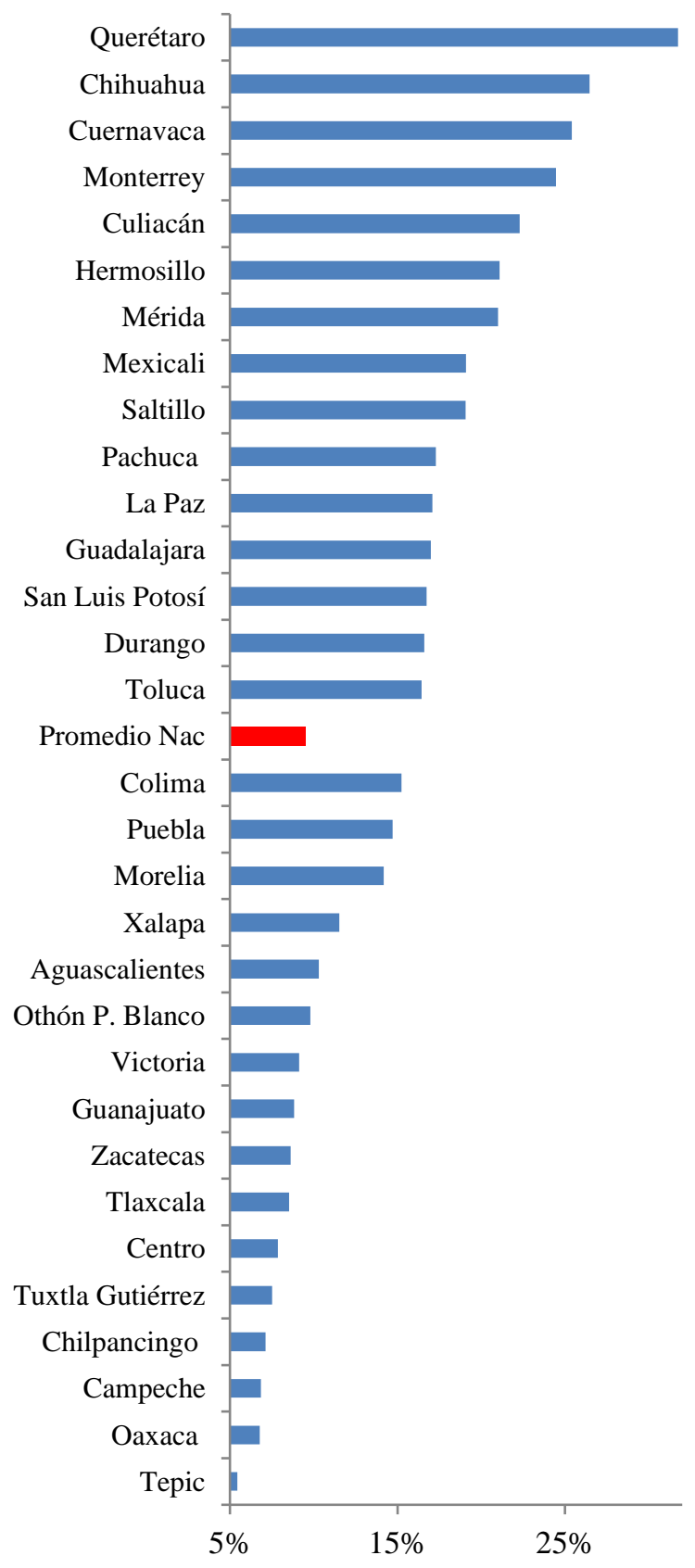

Gráfico 2 Ingresos fiscales locales con relación a los ingresos totales municipales promedio en el período 2008 $-2017$

Fuente: elaboración propia con datos de INEGI (2019) 
El impuesto predial constituye la fuente de ingresos propios más relevante para los municipios mexicanos.

En términos per cápita, durante el año 2017 se recaudaron, en promedio, \$ 419.0; sin embargo, mientras que Querétaro y Monterrey ingresaron $\quad \$ \quad 1,1123.0$ y $\$ \quad 1,034.0$ respectivamente, el municipio de Tepic solo reportó \$133.0 per cápita por concepto de recaudación de dicho tributo (ver Tabla 2).

\begin{tabular}{|l|r|}
\hline \multicolumn{1}{|c|}{ Municipio } & Predial per cápita (\$) \\
\hline Querétaro & $1,123.0$ \\
\hline Monterrey & $1,034.0$ \\
\hline Chihuahua & 702.0 \\
\hline Colima & 660.0 \\
\hline Guadalajara & 640.0 \\
\hline Culiacán & 596.0 \\
\hline Cuernavaca & 568.0 \\
\hline Mexicali & 517.0 \\
\hline Zacatecas & 507.0 \\
\hline Mérida & 498.0 \\
\hline Hermosillo & 479.0 \\
\hline La Paz & 447.0 \\
\hline Morelia & 447.0 \\
\hline Puebla & 428.0 \\
\hline Toluca & 398.0 \\
\hline Oaxaca & 373.0 \\
\hline Durango & 341.0 \\
\hline San Luis Potosí & 311.0 \\
\hline Pachuca & 306.0 \\
\hline Othón P. Blanco & 299.0 \\
\hline Aguascalientes & 293.0 \\
\hline Saltillo & 267.0 \\
\hline Tuxtla Gutiérrez & 239.0 \\
\hline Centro & 233.0 \\
\hline Campeche & 230.0 \\
\hline Xalapa & 211.0 \\
\hline Tlaxcala & 206.0 \\
\hline La Paz & 195.0 \\
\hline Victoria & 159.0 \\
\hline Chilpancingo & 140.0 \\
\hline Tepic & 133.0 \\
\hline & \\
\hline &
\end{tabular}

Tabla 2 Monto de recaudación por concepto de impuesto predial en términos per cápita en 2017

Fuente: elaboración propia con datos de SHCP (2019)

Finalmente, se reportan los resultados del análisis causal. Se encuentra que el coeficiente de determinación ajustado $\mathrm{R}^{2}$ es de 0.595 , esto es, en su conjunto, las variables exógenas permiten explicar el $59.5 \%$ de la varianza de la variable dependiente (impuestos).

\begin{tabular}{|l|r|r|r|}
\hline \multicolumn{1}{|c}{ Variable } & \multicolumn{1}{c}{ Coef } & \multicolumn{1}{c|}{ p-valor } \\
\hline C & 0.583 & 22.26 & 0.000 \\
\hline Part_fed & -0.622 & -14.75 & 0.000 \\
\hline Apor_fed_est & -0.552 & -14.62 & 0.000 \\
\hline Derechos & -0.286 & -3.07 & 0.002 \\
\hline Productos & -0.409 & -2.69 & 0.007 \\
\hline Aprovech & -0.154 & -1.70 & 0.089 \\
\hline Financ & -0.542 & -12.58 & 0.000 \\
\hline
\end{tabular}

Tabla 3 Resultados de las estimaciones

Fuente: elaboración propia

Con relación a las variables independientes, se encontró que cada una de ellas tiene una relación causal negativa y estadísticamente significativa con la variable explicada. Las magnitudes de los coeficientes de las variables explicativas permiten identificar la intensidad del impacto que cambios unitarios en éstas podrían ocasionar sobre la variable dependiente.

Respecto de los rubros de ingresos provenientes de fuentes externas, se encontró que un ajuste positivo de un millón de pesos en recursos obtenidos a través de participaciones federales generaría una disminución en los ingresos que se obtienen a través de la recaudación de impuestos locales por el orden de 622 mil pesos. Asimismo, se estima que por cada peso que aumenten los ingresos provenientes de aportaciones federales y estatales se estima una reducción de 55 centavos en el monto de los recursos fiscales municipales. También se localizó un impacto negativo en los ingresos fiscales locales de 542 mil pesos por cada millón de pesos originados a partir de la contratación de financiamientos o deuda pública.

Con relación a los ingresos propios de carácter no tributario, se encontró un efecto que aunque menor al provocado por los ingresos de origen externo, es negativo. Específicamente, por cada 100 mil pesos que ingresen vía productos, derechos y aprovechamientos se prevé una reducción de $\$ 40,900, \$ 28,600$ y $\$$ 15,400 respectivamente en los ingresos fiscales locales.

\section{Conclusiones}

Los ingresos públicos municipales reportan una marcada dependencia respecto de los recursos que los órdenes de gobierno estatal y federal les transfieren. 
El objetivo general del estudio es identificar la relación causal entre los diversos componentes que integran los ingresos públicos no tributarios de los municipios y los ingresos fiscales municipales en México. Se encontró que, en efecto, existe una correspondencia negativa entre el nivel de ingresos proveniente de fuentes externas como lo son las participación federales y las aportaciones federales y estatales con los ingresos generados a partir del ejercicio de las potestades tributarias municipales; esto representa un elemento de debate en torno al funcionamiento del Sistema Nacional de Coordinación Fiscal y los efectos que, sobre la generación de ingresos propios, ocasiona en el ámbito municipal.

También se identificó una asociación indirecta entre los recursos obtenidos mediante la contratación de deuda pública y los ingresos fiscales municipales; en este caso, el endeudamiento público podría estar generando circunstancias nocivas respecto de los incentivos que las administraciones públicas municipales podrían tener con relación a financiar programas, planes y proyectos con recursos propios.

Los ingresos propios no tributarios (productos, derechos y aprovechamientos) se comportaron en el mismo sentido que las fuentes de ingresos no propias pero con impactos menores sobre el nivel de recursos obtenidos mediante la recaudación de impuestos. Estos hallazgos permiten suponer que los gobiernos municipales podrían estar relajando su política recaudatoria en virtud del soporte financiero que representan los recursos provenientes de los estados y de la federación así como los ingresos originados a partir de la contratación de deuda pública.

Esta aparente ineficacia en el ejercicio de las potestades tributarias podría tener, en cierto modo, una connotación política. Es decir, con las reservas de las comprobaciones correspondientes, podría presumirse que los gobiernos locales tienden a perpetuar su pereza fiscal deliberadamente en virtud del desagrado generalizado que entre sus ciudadanos podría ocasionar un ajuste en la política recaudatoria local en cuanto a mecanismos de coacción o de ajustes en las tasas y valores de los bienes gravados con impuestos locales lo cual, sin duda, tendría.
Finalmente, pareciera que el pacto fiscal mexicano requiere una revisión integral que aborde, entre otras, la perspectiva de la generación de ingresos locales para fomentar una mayor intervención de los gobiernos subnacionales respecto de la descentralización fiscal en el ámbito de los ingresos y no solamente en el rubro del gasto en el cual, por cierto, si ha habido avances considerables con relación a la participación de los municipios en el gasto público total.

\section{Referencias}

Camberos, R., Jonathan, J., \& Poom Medina, J. (2013). Factores políticos e institucionales asociados a la deuda municipal en el noroeste de México: 1989-2007. Andamios, 10(21), 6193.

Chávez Maza, Luis Augusto. (2014). Desistimiento fiscal, incertidumbre y propiedad social en los municipios de México. Región y sociedad, 26(61), 87-117. Recuperado en 09 de septiembre de 2019, de http://www.scielo.org.mx/scielo.php?script=sci _arttext\&pid=S1870$39252014000400003 \& \operatorname{lng}=\mathrm{es} \& \operatorname{tlng}=\mathrm{en}$.

Chávez Maza, L. A. (2014). Desistimiento fiscal, incertidumbre y propiedad social en los municipios de México. Región y sociedad, 26(61), 87-117.

Cejudo, G., \& Ríos, A. (2009). La rendición de cuentas del gobierno municipal en México. Centro de Investigación y Docencia Económicas (CIDE).

Consejo Nacional de Armonización Contable (2019). Clasificador por rubro de ingresos. Recuperado de https://www.conac.gob.mx/work/models/CON AC/normatividad/NOR_01_02_001.pdf

Eguino, H., Perazzo, A., Pinto, F., Antonio Pérez, J., \& del Istmo Centroamericano, F. D. M. (2002). Ingresos municipales en Centroamérica: situación actual y perspectivas de modernización.

Espinosa, S., Martinez, J., \& Martell, C. (2018). ¿Por qué algunos municipios en México son mejores recaudadores de impuesto predial que otros? Gestión y política pública, 27(2), 375395. 
Instituto Nacional de Estadística y Geografía (2019). Estadística de Finanzas Públicas Estatales y Municipales. https://www.inegi.org.mx/programas/finanzas/d efault.html\#Tabulados

Londoño, A. L. G., \& Ossa, J. A. G. (2008). La gerencia pública local en Colombia. Un asunto de continuidad política en la administración pública municipal. Revista Ciencias Estratégicas, 16(19), 65-78.

Mandujano Ramos, N. (2010). Federalismo fiscal en México. Una propuesta para fortalecer la hacienda pública estatal. Instituto de Investigaciones Económicas, UNAM.

Núñez, J. (2005). Diagnóstico básico de la situación de los ingresos por impuestos del orden municipal en Colombia. Documento cede, 44, 1-137.

OECD (2018). Revenue Statistics 2018. OECD Publishing, Paris.

Secretaría de Hacienda y Crédito Público (2019). Transparencia presupuestaria. Datos abiertos. https://www.transparenciapresu puestaria.gob.mx/es/PTP/Datos_Abiertos

Sour, L. (2004). El sistema de transferencias federales en México: Premio o castigo para el esfuerzo fiscal de los gobiernos locales urbanos.

Unda Gutierrez, M., \& Moreno Jaimes, C. (2015). Property Tax Collection in Mexico: An Analysis of Its Economic Determinants in the period 1969-2010. Revista Mexicana de Ciencias Políticas y Sociales, 6(225), 45-77. 